\title{
Can proper motions of dark-matter subhalos be detected?
}

\author{
Shin'ichiro Ando, ${ }^{1}$ Marc Kamionkowski, ${ }^{1}$ Samuel K. Lee, ${ }^{1}$ and Savvas M. Koushiappas ${ }^{2}$ \\ ${ }^{1}$ California Institute of Technology, Mail Code 130-33, Pasadena, California 91125, USA \\ ${ }^{2}$ Department of Physics, Brown University, 182 Hope Street, Providence, Rhode Island 02912, USA
}

(Received 4 September 2008; published 4 November 2008)

\begin{abstract}
One of the goals of NASA's Fermi Gamma-ray Space Telescope (formerly GLAST) will be the detection of gamma rays from dark-matter annihilation in the Galactic halo. Theoretical arguments suggest that dark matter may be bound into subhalos with masses as small as $10^{-4}-10^{2} M_{\oplus}$. If so, it may be possible to detect individual subhalos as point sources in the Fermi Telescope. It has further been argued that some of these point sources may exhibit proper motions. Here we show that upper limits to the diffuse gamma-ray background constrain the number of subhalos close enough to exhibit proper motions to be less than one.
\end{abstract}

DOI: 10.1103/PhysRevD.78.101301

PACS numbers: 95.85.Pw, 95.35.+d, 98.35.Gi

\section{INTRODUCTION}

One of the major goals of the recently launched Fermi Gamma-ray Space Telescope (formerly GLAST) [1] is to detect dark-matter annihilation into gamma rays, as expected, with some flux, if dark matter is composed of weakly interacting massive particles (WIMPs), such as the neutralino in supersymmetric models [2]. Theoretical arguments suggest that dark matter in the Galaxy may be distributed in subhalos $[3,4]$ with masses that may extend all the way down to $10^{-4}-10^{2} M_{\oplus}$, if WIMPs make up dark matter [5].

References [6] pointed out that if WIMP substructure extends to such small scales, then it may be possible not only to detect individual subhalos as point sources with the Fermi Telescope, but also that up to ten of these point sources may exhibit proper motions.

In this paper, we revisit this proposal. We use the diffuse gamma-ray background measured with the Energetic Gamma-Ray Experimental Telescope (EGRET) [7] to place an upper limit to the gamma-ray luminosity of these subhalos (see also Ref. [8]). This constraint then limits the number of detectable subhalos that are sufficiently close to exhibit proper motions. A similar argument was also made in Ref. [9] for a specific numerically simulated subhalo model. Here, we present an analytic and fully general argument. The largest number of detectable proper motions is obtained if the subhalo mass distribution is sharply peaked around $10^{-3}-10^{-2} M_{\odot}$, and even then, the expected number of detectable proper motions is less than 1 .

Before proceeding with the detailed calculation, we begin in Sec. II with an order-of-magnitude estimate. Section III then describes more precisely the EGRET upper limit to the diffuse background and the constraints implied for the subhalo population. We then evaluate in Sec. IV the number of detectable proper motions, first by assuming all subhalos have the same mass, and then by generalizing to the more realistic case of a power-law distribution of subhalo masses. In Sec. V, we discuss the results and how they vary upon changing the assumptions taken from their canonical values adopted in the main body of the paper.

\section{ORDER-OF-MAGNITUDE ESTIMATE}

If the Fermi Telescope can detect proper motions as small as $0.1^{\circ}$ and subhalos have a transverse velocity $\sim 200 \mathrm{~km} \mathrm{~s}^{-1}$, then a subhalo must be within a distance $d_{\mathrm{pm}} \sim 0.35 \mathrm{pc}$ to have a detectable proper motion over three years. If the point-source flux sensitivity is $F_{\text {sens }}$, a source with a detectable proper motion must have a luminosity $L \sim 4 \pi d_{\mathrm{pm}}^{2} F_{\text {sens. }}$. Each detected proper motion thus implies a subhalo contribution to the luminosity density of order $L / V \sim 3 F_{\text {sens }} / d_{\mathrm{pm}}$. This implies a total luminosity, integrated over the volume of the Milky Way interior to the solar radius, of $L_{\mathrm{MW}} \gtrsim 4 \pi r_{\odot}^{3} F_{\text {sens }} / d_{\mathrm{pm}}$, where $r_{\odot}=$ $8.5 \mathrm{kpc}$ is our distance from the Galactic center (the " $Z$ " arises because the halo density most generally decreases with radius). Thus, a flux from the Milky Way of $F_{\mathrm{MW}} \sim L_{\mathrm{MW}} /\left(4 \pi r_{\odot}^{2}\right) \gtrsim\left(r_{\odot} / d_{\mathrm{pm}}\right) F_{\text {sens }}$ is implied for each proper motion detected. This is a large flux-larger than upper limits even for one detected proper motion-as the more detailed calculations below will show.

\section{EGRET CONSTRAINTS TO THE SUBHALO POPULATION}

Assume that a fraction $f$ of the dark matter in the Galactic halo is composed of subhalos of mass $M$ and luminosity $L,{ }^{1}$ and we define a mass-to-gamma-rayluminosity ratio $Y \equiv M / L$. Assume further that the radial distribution of subhalos follows the density profile $\rho(r)$ of the Milky Way halo.

\footnotetext{
${ }^{1}$ Throughout this paper, the luminosity is the number (not energy) of photons emitted per unit time; similarly, we deal with number fluxes and intensities.
} 
The gamma-ray intensity $I(\psi)$ (units of photons $\mathrm{cm}^{-2} \mathrm{~s}^{-1} \mathrm{sr}^{-1}$ ), due to gamma rays from these subhalos, is

$$
I(\psi)=\frac{f}{4 \pi \Upsilon} \int d l(\psi) \rho(r[l(\psi)]),
$$

along a line of sight at an angle $\psi$ from the Galactic center. Here, $l$ is the distance along the line of sight $\psi$; i.e., $r^{2}=$ $r_{\odot}^{2}+l^{2}-2 r_{\odot} l \cos \psi$.

For $\rho(r)$, we use the Navarro-Frenk-White (NFW) [10] profile,

$$
\rho(r)=\frac{\rho_{s}}{\left(r / r_{s}\right)\left(1+r / r_{s}\right)^{2}},
$$

where $\rho_{s}=5.4 \times 10^{-3} M_{\odot} \mathrm{pc}^{-3}$ is the characteristic density and $r_{s}=21.7 \mathrm{kpc}$ is the scale radius. (These numerical values, taken to explain the virial mass and rotation curves [11], are adopted for illustrative purposes, but the main conclusions do not depend on them.) This gives $\rho_{\odot}=$ $7 \times 10^{-3} M_{\odot} \mathrm{pc}^{-3}$ as the local density.

The diffuse gamma-ray background measured with EGRET places an upper limit to $f \Upsilon^{-1}$. If the subhalo gamma rays form a continuum spectrum, then we use the diffuse gamma-ray-background intensity measured with EGRET [12] at $\psi=90^{\circ}$ as a conservative upper limit to $I(\psi)$ from subhalos (roughly the same bound obtains for $\left.\psi=180^{\circ}\right)$. For line emission, on the other hand, we approximate the upper limit to the gamma-ray-line intensity, averaged over a $10^{\circ} \times 10^{\circ}$ region around the Galactic center, by $2 \times 10^{-6}(E / \mathrm{GeV})^{-1 / 2} \mathrm{~cm}^{-2} \mathrm{~s}^{-1} \mathrm{sr}^{-1}$ over the energy range $0.1 \mathrm{GeV}<E<10 \mathrm{GeV}$ [13]. (Scaling our results to more conservative limits on line intensity [14] is trivial.) At an energy $E=10 \mathrm{GeV}$, the upper limits to both the continuum and line intensities turn out, coincidentally, to be

$$
f \Upsilon^{-1}=10^{29} I_{*} M_{\odot}^{-1} \mathrm{~s}^{-1},
$$

where $I_{*} \leq 1$ is the fractional contribution from subhalos to the gamma-ray-background intensity, the total being due, additionally, to traditional astrophysical sources and/ or WIMP annihilation in the $1-f$ fraction of the Milky Way halo that is smoothly distributed. We discuss further this assumption later on.

\section{PROPER-MOTION DETECTION}

Suppose that a dark-matter subhalo moves transverse to the line of sight with a velocity $200 \mathrm{~km} \mathrm{~s}^{-1}$. Then, in a three-year experiment, the subhalo will move an angular distance of $0.1^{\circ} \theta_{0.1}$ if it is at a distance

$$
d_{\mathrm{pm}} \equiv 0.35 \mathrm{pc} \theta_{0.1}^{-1} \text {. }
$$

Here, we take the minimum proper motion detectable by the Fermi Telescope to be $0.1^{\circ}$ (for $10 \mathrm{GeV}$ gamma rays) as a canonical value [15], but keep the $\theta_{0.1}$ dependence in the following. Thus, a proper motion can be detected only if the subhalo is closer than $d_{\mathrm{pm}}$.

\section{A. The monoluminous case}

First, consider the case where the background is due to subhalos of a one mass scale $M$. We call this the monoluminous case. The number of subhalos within a distance $r$ of the Earth is

$N_{\mathrm{sh}}(<r)=\frac{4 \pi r^{3}}{3} \frac{f \rho_{\odot}}{M}=1.3 \times 10^{-3} \theta_{0.1}^{-3}\left(\frac{r}{d_{\mathrm{pm}}}\right)^{3}\left(\frac{M}{f M_{\odot}}\right)^{-1}$.

For the source to be detected at $d_{\mathrm{pm}}$, the luminosity $L=$ $\Upsilon^{-1} M$ has to be larger than $4 \pi d_{\mathrm{pm}}^{2} F_{\text {sens }}$, where $F_{\text {sens }}$ is the point-source flux sensitivity; for the Fermi Telescope, we take $F_{\text {sens }} \equiv 10^{-10} F_{10} \mathrm{~cm}^{-2} \mathrm{~s}^{-1}$ at $10 \mathrm{GeV}$, with $F_{10} \simeq 1$ [15]. With the EGRET constraint to $Y$ [Eq. (3)], the condition for detection at $d_{\mathrm{pm}}$ is $M \geq M_{\mathrm{pm}}$, where

$$
M_{\mathrm{pm}} \equiv 1.5 \times 10^{-2} f M_{\odot} F_{10} I_{*}^{-1} \theta_{0.1}^{-2} .
$$

If $M<M_{\mathrm{pm}}$, the subhalo can only be detected up to a distance $\left[\Upsilon^{-1} M /\left(4 \pi F_{\text {sens }}\right)\right]^{1 / 2}<d_{\mathrm{pm}}$.

Thus, the number of detectable proper motions is

$$
\begin{aligned}
N_{\mathrm{pm}} & = \begin{cases}N_{\mathrm{sh}}\left(<d_{\mathrm{pm}}\right), & \text { for } M \geq M_{\mathrm{pm}}, \\
N_{\mathrm{sh}}\left(<\left[\frac{\mathrm{Y}^{-1} M}{4 \pi F_{\mathrm{sens}}}\right]^{1 / 2}\right), & \text { for } M<M_{\mathrm{pm}},\end{cases} \\
= & 0.09 F_{10}^{-1} I_{*} \theta_{0.1}^{-1} \times \begin{cases}\left(\frac{M}{M_{\mathrm{pm}}}\right)^{-1}, & \text { for } M \geq M_{\mathrm{pm}}, \\
\left(\frac{M}{M_{\mathrm{pm}}}\right)^{1 / 2}, & \text { for } M<M_{\mathrm{pm}},\end{cases}
\end{aligned}
$$

To summarize, assuming the diffuse-background density measured by EGRET, and assuming that this background is due to annihilations in subhalos, the maximum probability to detect proper motion in a nearby subhalo is $\sim 10 \%$. This probability is maximized for subhalo masses $M \sim$ $10^{-2} f M_{\odot}$. Larger-mass subhalos will provide a larger number of detectable point sources (that can be detected to larger distances), but fewer with proper motions. On the other hand, there will be more nearby objects if they are less massive, but they will be too faint to be detectable.

\section{B. Power-law mass and luminosity functions}

Now suppose, as before, that a fraction $f$ of the mass of the halo is in subhalos, but now assume that the masses are distributed with a power-law mass function,

$$
\frac{d n}{d M}(r, M)=\frac{f \rho(r)}{\ln \Lambda} M^{-2},
$$

with power-law index -2 , as suggested by numerical simulations [4]. Here, $\Lambda \equiv M_{\max } / M_{\min }$, and $M_{\min }$ and $M_{\max }$ are the lower and upper mass limits. If the population has a constant mass-to-luminosity ratio $Y$, then the bound in Eq. (3) still applies. 
The differential number $d N_{\mathrm{sh}} / d \ln M$ of subhalos per logarithmic mass interval within a distance $r$ from the Earth is given by Eq. (5), with the replacement $f \rightarrow$ $f / \ln \Lambda$. The simulations of Refs. [4] suggest $f / \ln \Lambda=$ 0.0064 ; typical values are $\ln \Lambda=35$ and $f \approx 0.2$. The differential number of subhalos with detectable flux and proper motions per logarithmic mass interval is then

$$
\begin{aligned}
\frac{d N_{\mathrm{pm}}}{d \ln M}= & 0.002 F_{10}^{-1} I_{*} \theta_{0.1}^{-1}\left(\frac{\ln \Lambda}{35}\right)^{-1} \\
& \times \begin{cases}\left(\frac{M}{M_{\mathrm{pm}}}\right)^{-1}, & \text { for } M \geq M_{\mathrm{pm}}, \\
\left(\frac{M}{M_{\mathrm{pm}}}\right)^{1 / 2}, & \text { for } M<M_{\mathrm{pm}} .\end{cases}
\end{aligned}
$$

Again the largest contribution comes from subhalos with masses around $M_{\mathrm{pm}}$. By integrating this expression over $\ln M$, we have

$$
\begin{aligned}
N_{\mathrm{pm}} \approx & 0.002 F_{10}^{-1} I_{*} \theta_{0.1}^{-1}\left(\frac{\ln \Lambda}{35}\right)^{-1} \\
& \times \begin{cases}\left(\frac{M_{\min }}{M_{\mathrm{pm}}}\right)^{-1}, & \text { for } M_{\mathrm{pm}} \leq M_{\min }, \\
3, & \text { for } M_{\min } \ll M_{\mathrm{pm}} \ll M_{\max },\end{cases}
\end{aligned}
$$

which is far smaller than in the monoluminous case. This is easily understood: Detectable proper motions still come from subhalos with masses around $M_{\mathrm{pm}}$. However, there are now fewer such subhalos, since the subhalo masses are distributed over a wider range. The contribution from $M_{\mathrm{pm}}$ is thus smaller by a logarithmic factor $\ln \Lambda$. Although we focused only on the case of $d n / d M \propto M^{-2}$ here, the argument applies to any mass function. Again, the largest number of detectable proper motions comes when the mass distribution is sharply peaked around $M_{\mathrm{pm}}$.

\section{DISCUSSION AND CONCLUSIONS}

In this short paper, we revisit the detectability of proper motions of dark-matter subhalos. Our main results are: (1) The EGRET diffuse-background constraint on the subhalo mass-to-luminosity ratio severely restricts the expected number of proper-motion detections with the Fermi Telescope to be less than 1. This argument does not rely on the details of any particle-physics model, in contrast to the earlier studies [6]. (2) The number of detectable proper motions is maximized if the subhalo mass distribution is sharply peaked around a mass $M_{\mathrm{pm}} \sim$ $0.01 f M_{\odot}$. In this case, the probability to detect one subhalo with a proper motion is $\sim 10 \%$. (3) This maximal probability is obtained only when dark-matter annihilation from subhalos dominates the diffuse gamma-ray background (i.e., $I_{*} \simeq 1$ ), and it is reduced in proportion to the fraction $I_{*}$ of the diffuse background due to subhalos. This fraction is likely to be much less than 1, as there may be significant contributions to EGRET's diffuse background from unresolved astrophysical sources and perhaps from WIMP annihilation in a smoothly distributed halo component. (4) If the subhalo mass function follows a power-law mass function, the expected number of proper-motion detections is further suppressed by a logarithmic factor $\ln \left(M_{\max } / M_{\min }\right)$, where $M_{\max }$ and $M_{\min }$ are masses of the largest and smallest subhalos. This factor could be $\sim 30-40$, in which case the chance of detection would be no greater than $1 \%$. In this calculation, we have used a canonical gamma-ray energy of $E=10 \mathrm{GeV}$. The results can be scaled (but will not differ too much) by taking into account the $E^{-1 / 2}$ dependence of $I_{*}$ and a roughly similar energy dependence of the Fermi Telescope point-source sensitivity $F_{10}$.

Under these assumptions, the main result is that the probability of detecting proper motion in subhalos is less than one. It is natural then to ask under what conditions proper motions can be detected. There are two parameters in our formalism which can be varied in order to explore this possibility.

The quantity $I_{*}$ can be used to parametrize the effects of halo-modeling uncertainties. If $\rho(r)$ increases less rapidly toward the Galactic center than in the NFW profile, then the predicted intensity will decrease and the bound in Eq. (3) is weakened (for line radiation, which is bound by the Galactic center) accordingly. If, for example, we postulate an extreme scenario where the halo density $\rho(r)$ is constant interior to the solar radius (holding the local density fixed), then the bound in Eq. (3) is weakened by about a factor of 4 . Likewise, $I_{*}$ can also quantify the effect of a galactocentric-radius-dependent fraction $f$. It may be that subhalos are more effectively disrupted closer to the Galactic center, and if so, then we should replace the fraction $f$ in Eq. (1) by a function $f(r)$ that decreases monotonically as $r \rightarrow 0$ and move $f(r)$ into the integrand. If we guess $f(r)=f_{0}\left(r / r_{\odot}\right)^{\alpha}$, we then find that for $\alpha=1$, the bound in Eq. (3) (with the replacement $f \rightarrow f_{0}$ ) is weakened by about a factor of 4 (the bound is weakened further by a factor of 6 if $\alpha=2$ ). Thus, if $f$ has a radial dependence, then its effects can be taken into account by allowing an increase in $I_{*}$ by a factor of 4 (for $\alpha=1$ ) to 6 (for $\alpha=2$ ). Note that this caveat applies only for line radiation (which we have bound by an upper limit to the gamma-ray flux from the Galactic center), but not to a continuum, for which we have used diffuse-background upper limits away from the Galactic center.

The results are sensitive to the angular resolution and duration of the experiment. Throughout this calculation we assumed a threshold for proper-motion detection of $0.1^{\circ}$ and a time scale of three years. The number of detectable proper motions increases as $\theta_{0.1}^{-1}$ [see Eqs. (7) and (10)]. Lowering the threshold for proper-motion detection in a fixed time scale can be possible if a nearby subhalo is 
present and detected at a high signal-to-noise ratio. In such a case the angular resolution of the instrument will improve as $\sim N_{\gamma}^{-1 / 2}$, where $N_{\gamma}$ is the number of photons detected from the source. In addition, increasing the time scale of the experiment from three to, e.g., 10 years (projected lifetime of the Fermi Telescope) will increase the probability of detection, as proper motion is linear with time.

In summary, unless the distribution of subhalos within the solar radius is severely suppressed and the Fermi
Telescope operates for at least 10 years, the expected number of proper-motion detections is less than one.

\section{ACKNOWLEDGMENTS}

We thank John Beacom for helpful comments. Work at Caltech was supported by the Sherman Fairchild Foundation (S. A.), DOE No. DE-FG03-92-ER40701, and the Gordon and Betty Moore Foundation. S. M. K. acknowledges support from Brown University.
[1] http://fermi.gsfc.nasa.gov.

[2] G. Jungman, M. Kamionkowski, and K. Griest, Phys. Rep. 267, 195 (1996); G. Bertone, D. Hooper, and J. Silk, Phys. Rep. 405, 279 (2005); D. Hooper and S. Profumo, Phys. Rep. 453, 29 (2007).

[3] S. Ghigna, B. Moore, F. Governato, G. Lake, T. Quinn, and J. Stadel, Mon. Not. R. Astron. Soc. 300, 146 (1998); A. A. Klypin, A. V. Kravtsov, O. Valenzuela, and F. Prada, Astrophys. J. 522, 82 (1999); A. A. Klypin, S. Gottlöber, A. V. Kravtsov, and A. M. Khokhlov, Astrophys. J. 516, 530 (1999); B. Moore, S. Ghigna, F. Governato, G. Lake, T. Quinn, J. Stadel, and P. Tozzi, Astrophys. J. 524, L19 (1999); A. Helmi, S. D. M. White, and V. Springel, Phys. Rev. D 66, 063502 (2002); D. Reed, F. Governato, T. Quinn, J. Gardner, J. Stadel, and G. Lake, Mon. Not. R. Astron. Soc. 359, 1537 (2005); A. Loeb and M. Zaldarriaga, Phys. Rev. D 71, 103520 (2005); A. M. Green, S. Hofmann, and D. J. Schwarz, Mon. Not. R. Astron. Soc. 353, L23 (2004); J. Cosmol. Astropart. Phys. 08 (2005) 003; J. Diemand, B. Moore, and J. Stadel, Nature (London) 433, 389 (2005); J. Diemand, M. Kuhlen, and P. Madau, Astrophys. J. 649, 1 (2006); C. Giocoli, L. Pieri, and G. Tormen, arXiv:0712.1476; V. Berezinsky, V. Dokuchaev, and Y. Eroshenko, Phys. Rev. D 77, 083519 (2008); M. Kamionkowski and S.M. Koushiappas, Phys. Rev. D 77, 103509 (2008).

[4] J. Diemand, M. Kuhlen, and P. Madau, Astrophys. J. 657,
262 (2007); 667, 859 (2007).

[5] X.1. Chen, M. Kamionkowski, and X.m. Zhang, Phys. Rev. D 64, 021302 (2001); S. Profumo, K. Sigurdson, and M. Kamionkowski, Phys. Rev. Lett. 97, 031301 (2006); T. Bringmann and S. Hofmann, J. Cosmol. Astropart. Phys. 04 (2007) 016.

[6] S. M. Koushiappas, Phys. Rev. Lett. 97, 191301 (2006); S. M. Koushiappas, AIP Conf. Proc. 921, 142 (2007); B. Moore, J. Diemand, J. Stadel, and T. R. Quinn, arXiv: astro-ph/0502213.

[7] http://heasarc.gsfc.nasa.gov/docs/cgro/egret.

[8] T. Oda, T. Totani, and M. Nagashima, Astrophys. J. 633, L65 (2005).

[9] L. Pieri, G. Bertone, and E. Branchini, Mon. Not. R. Astron. Soc. 384, 1627 (2008).

[10] J. F. Navarro, C. S. Frenk, and S. D. M. White, Astrophys. J. 490, 493 (1997).

[11] N. Fornengo, L. Pieri, and S. Scopel, Phys. Rev. D 70, 103529 (2004).

[12] P. Sreekumar et al. (EGRET Collaboration), Astrophys. J. 494, 523 (1998).

[13] A. R. Pullen, R. R. Chary, and M. Kamionkowski, Phys. Rev. D 76, 063006 (2007).

[14] G. D. Mack, T. D. Jacques, J. F. Beacom, N. F. Bell, and H. Yuksel, Phys. Rev. D 78, 063542 (2008).

[15] http://www-glast.slac.stanford.edu. 